\title{
Rapid Detection of Gene HLA-A3101 on Membrane Based Lateral-Flow Strips
}

\author{
Jui-Chuang $\mathrm{Wu}^{*}$, Yu-Shiun Huang \\ Department of Chemical Engineering, Chung Yuan Christian University, Taiwan, R.O.C. \\ * Corresponding author. Tel.: 886-32654141; email: ray_j_wu@cycu.edu.tw \\ Manuscript submitted January 10, 2018; accepted March 8, 2018. \\ doi: 10.17706/ijbbb.2018.8.4.218-225
}

\begin{abstract}
Gene Human leukocyte antigen (HLA)-A3101 is known responsible of a medicine allergy which may cause a fatal outcome. General clinics need a quick test to determine if a patient is engaged with the related gene defect and avoid the medical prescription from suspicious drugs. In this study, Gene HLA-A3101 is detected on membrane-based lateral-flow (MBFL) strips, incorporated with an immune reaction and color readouts developed by gold nanoparticles. The HLA-A-3101 DNA modified with biotin and digoxigenin labels was amplified by the Polymerase Chain Reaction (PCR) and then detected on the MBFL strips. The optimal condition of antibody concentration was at least $10 \mu \mathrm{g} / \mathrm{ml}$, to avoid the antibody-gold complex from aggregation. The signal can be observed as low as $0.49 \mathrm{ng}$ of the PCR product by naked eyes. The specificity of PCR primers was also investigated their annealing validity. The detection specificity from other species genes, including a plant, a virus, and a human gene, were also verified. The coefficient of variation values of the intra assay, which tests the same sample in the same day, and the inter assay, which tests three samples in three consecutive days, are in satisfactory ranges of $4.6 \%$ and $6.5 \%$, respectively.
\end{abstract}

Key words: Gene HLA-A3101, membrane based lateral-flow detection, gold nanoparticles, medicine allergy.

\section{Introduction}

Human leukocyte antigens (HLA) are responsible with many human self-immune diseases [1]. Recent researches indicate that several HLA allele genes may cause adverse drug reactions (ADR's) [2] and drug-induced liver injury (DILI) [3]. These pairs of HLA genes and their corresponding allergic medicines include HLA-B5701/Abacavir [4], HLA-B5801/Allopurinol [5], [6], HLA-A3101/Carbamazepine [3], and so on. This study will develop a membrane-based lateral-flow (MBLF) test to perform a rapid detection on HLA-A3101.

The MBLF test is one of the most efficient diagnosis tools for public health at care sites. Starting from the rapid detection on human chorionic gonadotropin (HCG) in a urine sample to determine if a woman is pregnant, the MBLF test has been widely applied in different fields. These applications cover the detections of pesticide residue in agriculture products [7]-[9], drug abuses [10], health care in husbandry [11], [12], and so on.

The MBLF technology has ever been also applied on the detection of various Polymerase Chain Reaction (PCR) products, for instances, allergens [13], [14], bacteria [15], pathogens [16], antibodies [17], and antigens [18], [19]. Our recent study revealed that an electric field could enhance the detection performance of single-trended Deoxyribonucleic acids (DNA) on the MBLF strips [20]. 
The MBLF offers lots of advantages in medical early diagnoses. It provides a cost-effective platform to manipulate samples. Secondly, it gives quick detection readouts without instrumental aids and a flexible temperature range for the application. Since the test operators do not need much training, homecare and clinics are its ideal application sites.

This study adopts the MBLF test to detection HLA-A3101 by labeling two ligands on its PCR products. One ligand is recognized by the report antibody loaded on the conjugate pad of the MBLF strip, and the other by the capture reagent on the capturing zone. The gold nanoparticles serve as the readout color, which can be directly read by naked eyes.

The detection principle of this assay is shown as Figure 1. The HLA3101 gene is first amplified by a PCR with labeling two ligands. This PCR product is then applied on the sample pad. As it flows through the conjugate pad, which is deposited with the report antibody, the PCR product will interact with the report antibody, since they have an affinity with each other. After the immune reaction, the PCR product-antibody complex flows ahead through the nitrocellulose membrane by a capillary force induced by the porous membrane material of nitrocellulose, of which porosity is around $5 \mu \mathrm{m}$. At the capture zone, the complex is again recognized on the other ligand by the capturing reagent deposited in advance on the membrane to form the test line. A readout color will appear on the capture zone to indicate a positive result, otherwise negative. An absorbent pad is placed at the other end of the strip to absorb excess fluid and keeps the flow continuous.

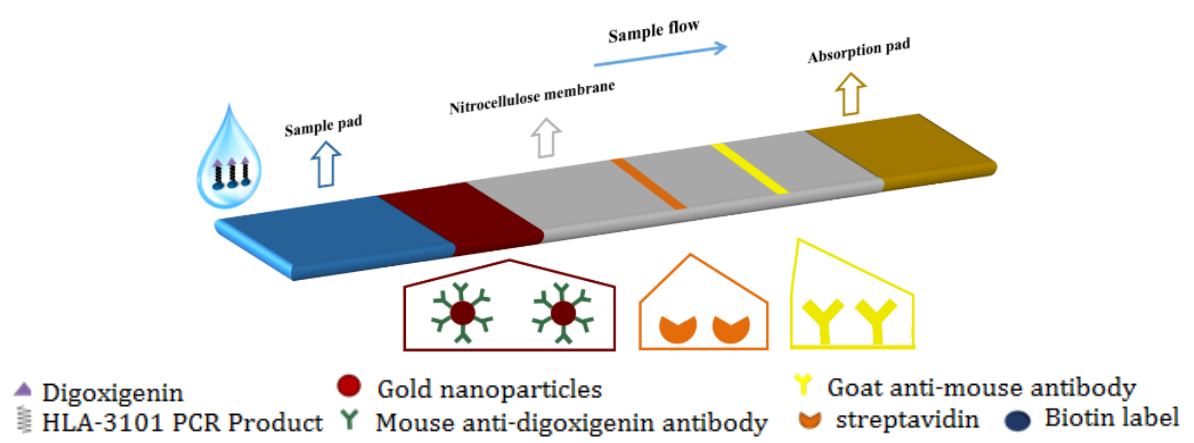

Fig. 1. The detection principle of the MBLF on HLA3101. Gene HLA3101's PCR product is induced to laterally flow through the membrane and encounters the report antibody and the capturing reagent, which respectively recognize tow ligands labeled on the PCR product. The result is identified by the gold color appearing or not at the capture zone as the test line.

\section{Experimental}

\subsection{Materials}

HLA-A3101 template (GenBank accession number: M84375.1) has a length of $124 \mathrm{bp}$. Its forwarding and reversing primers were 5'-digoxigenin-GTTCTCACACC ATCCAGATG-3' and 5'-bioten-GAGCGCAGGTCCT CGTTCAA-3', respectively. The ligands digoxigenin and biotin were respectively labeled on two primers at the $5^{\prime}$ ends.

For the specificity test, human genes of HLA-B5801 and PSMA5, plant gene of Arabidopsis thaliana Pda13015, Avian Influenza gene of H5, and their corresponding primers were also employed. The human gene PSMA5 is a popular genetic model. It was recently reported that it exists mainly as a tetramer [21]. All templates and PCR primers were synthesized by Biokit Biotech (Taiwan).

\subsection{Report and Control Antibodies}

Mouse anti-digoxigenin IgG antibody serviced as the report antibody. This monoclonal antibody was 
labeled with 30nm gold particles before being deposited on the conjugate pad.

Goat anti-mouse IgG antibody served as the control antibody and was directly deposited on the control zone, which was just next to the capture zone by $5 \mathrm{~mm}$ apart. If the control line does not appear, the test is invalid and needs to run again. Both antibodies were purchased from Biomate (Taiwan).

\subsection{Elements of MBLF Strips}

The nitrocellulose membrane with $5 \mu \mathrm{m}$ porosity served as the detection platform. Nitrocellulose has an excellent affinity with protein and provides a permanent binding after 24-hr post-dispensing desiccation in a 40\%RH dry box. The membrane was first adhered onto a adhesive plastic card and then deposited with the capture reagent and the control antibody.

The sample pad served as a sample reservoir to slowly release sample onto the conjugate pad. The conjugate pad, right next to the sample pad with a partially overlapping, was deposited with the gold-labeled report antibody. The absorbent pad was placed at the other end of the strip to provide a continuous capillary flow induced by the absorption of excess sample fluid from the pad. All membrane and pad elements were purchased from Rega (Taiwan).

\subsection{Other Reagents}

Streptavidin, which is deposited on the membrane as the test line, serves as the capturing reagent to capture biotin ligand labeled on the PCR product. If a red-color line appears at the capturing zone, the result is identified as positive, otherwise negative.

Nano-gold particles serve as the readout indicator. Its size is about $40 \mathrm{~nm}$ and appears in red. Rega (Taiwan) offered this commercial product.

\subsection{Instrument}

AgitestTM RP-1000 (Rega, Taiwan) served as the antibody and reagent dispenser to plot lines on the designated zones. Strip cutter (Jihshuenn JS-101, Taiwan) cut pad-membrane assembly into 5mm-wide strips. PCR machine (Astec PC-320, Japan) was used as the gene amplifier. Gel electrophoresis (BioBasic, Mupid-exU, Canada) was performed to verify the PCR validation. The gel electrophoresis was read at GeneFlash (Syngene, USA). The concentrations of antibody were measured by NanoDrop 1000.

\subsection{Procedures}

Before printing the control antibody (control line) and the capturing reagent (test line) on the membrane, the nitrocellulose membrane was first adhered on a plastic card. The test line is designed to be within $2 \sim 2.5 \mathrm{~cm}$ from the sample application zone and the control line is $5 \mathrm{~mm}$ apart from the test line. After printing finished, the plastic card is preceded for adhering other three pads: the sample, conjugate and absorbent pads. Before this adhesion step, the report antibody is deposited on the conjugate pad and dried at $37^{\circ} \mathrm{C}$ for $2 \mathrm{hr}$. The finished membrane-pad-card assembly is finally cut into $5 \mathrm{~mm}$-wide and ready-to-use strips.

For detections on PCR products for a typical run, $3 \mu \mathrm{l}$ of the sample was added into $67 \mu \mathrm{l}$ of PBS buffer and the mixed solution was then added onto the sample pad. The room humidity was set at $40 \% \mathrm{RH}$ and $26^{\circ} \mathrm{C}$. High room humidity causes moisture deposition from the atmosphere onto nitrocellulose membrane and slowing down the lateral flow of fluid. The detection time will be then delay. The sample-antibody complex will also be retarded on membrane to form a signal background.

The optimal concentration and $\mathrm{pH}$ of mouse anti-digoxigenin antibody for nano-gold conjugation were set at $10 \mu \mathrm{g} / \mathrm{ml}$ and 8.6 , respectively. Other $\mathrm{pH}$ conjugation conditions will cause gold-particle aggregation. As a nano-size material is aggregated, it loses its original physical property. In our case, the nano-gold 
particles became dark purple and smeared the membrane if they are forced to apply.

\section{Result and Discussion}

\subsection{Validation of PCR Annealing Temperature}

The general formula [22] for the optimal PCR's annealing temperature is

$$
T_{m}=81.5+16.6 \log \frac{[\text { salt }]}{1+0.7[\text { salt }]}+41 * G C \%-500 / L .
$$

Therefore, $59 \pm 3^{\circ} \mathrm{C}$ was set as the PCR condition, and the products were detection on MBLF strips after PCR was complete. As shown in Fig. $2,59^{\circ} \mathrm{C}$, obtained from the formula, gave the best performance.

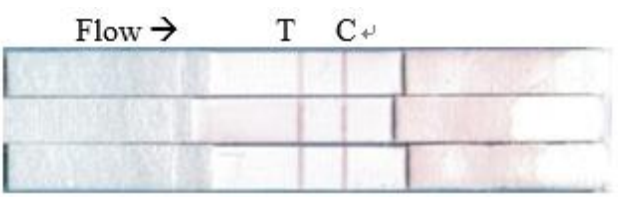

Fig. 2. Optimization of the PCR's annealing tempearture. From top to down, 62,59 , and $56^{\circ} \mathrm{Cwere}$ set.

\subsection{Verification of PCR Products}

The PCR products were verified by running 2\% agarose gel electrophoresis to see if they were successfully amplified. As shown in Figure 3, PCR products of HLA-A3101 (124bp), HLA-Cw1602 (264bp), Avian Influenza H5 (358bp), human PSMA5 (433bp), and Arabidopsis thaliana Pda13015 (252bp) were all located at their size-corresponding positions of the gel, indicating all PCR products were correctly amplified.

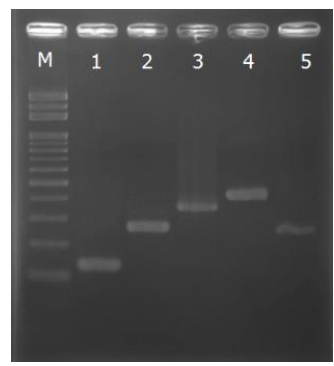

Fig. 3. Agarose gel electrophoresis to verify the PCR products. Lane M: the ladder, 1: HLA-A3101(124bp); 2: HLA-Cw1602(264bp); 3: H5(358bp); 4: PSMA5(433bp); 5: Pda13015(252bp).

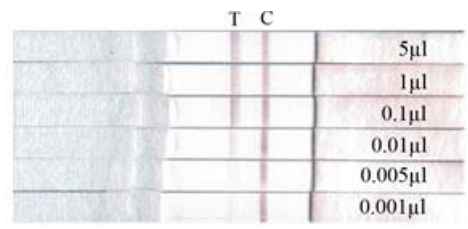

Fig. 4. The detection limit of HLA-A3101 PCR product on MBLF strips. The detection signal can be recognized as low as $0.01 \mu l$.

\subsection{Detection Limit of HLA-A3101 on MBLF Strips}

Different volumes of HLA-A3101 PCR product were added onto MBLF strips in $5 \mu \mathrm{l}, 1 \mu \mathrm{l}, 0.1 \mu \mathrm{l}, 0.01 \mu \mathrm{l}$, $0.005 \mu \mathrm{l}$, and $0.001 \mu \mathrm{l}$. The result is shown in Figure 4, the detection limit was as low as $0.01 \mu \mathrm{l}$, and of which 
corresponding amount was about $0.49 \mathrm{ng}$. Since the real detection limit will actually depend on the sampling gene template concentration during the application, the result here only provides a reference for the condition staring from 2ng of gene template mixed into total $50 \mu \mathrm{l}$ of PCR 25-cycle batch.

This result was also scanned their optical density to digitize the eye readings as shown in Fig. 5.

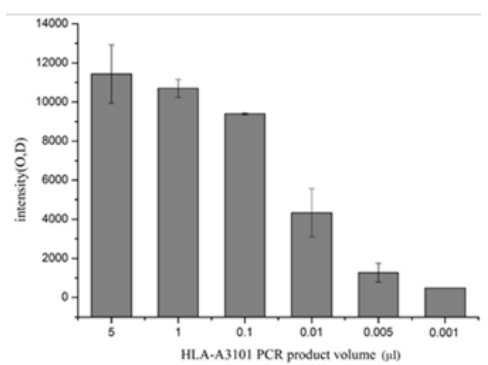

Fig. 5. Digitized plot for the detection limit of HLA-A3101 PCR product on MBLF strips. The detection signal threshold can be recognized at around 4000 OD in our analysis approach.

\subsection{Detection Specificity of HLA-A3101 on MBLF Strips}

The primers of HLA-A3101 were added to other four genes' PCR batches: HLA-Cw1602 (264bp), H5 (358bp), PSMA5 (433bp), and Pda13015 (252bp) to see if a non-specific PCR amplification occurred. Figures 6 to 8 show their PCR products run on MBLF strips and agarose gel.

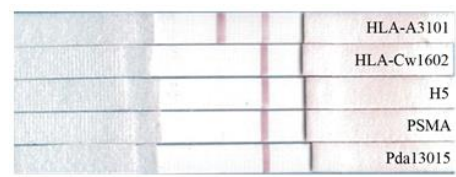

Fig. 6. Specification test of HLA-A3101 primers with other gene templates on MBLF strips. HLA-A3101's primers show a loyalty to their own template.

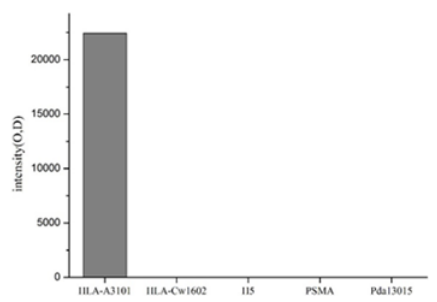

Fig. 7. Digitized plot of the Specification test of HLA-A3101 primers with other gene templates on MBLF strips.

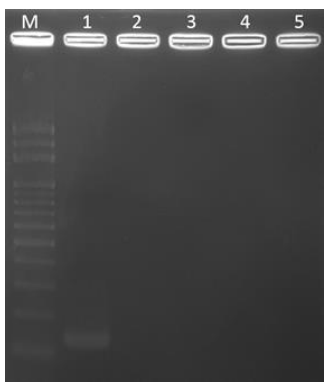

Fig. 8. Agarose gel electrophoresis for the specification test. Lane M: the ladder; HLA-A3101 primers run with Lane 1: HLA-A3101; 2: HLA-Cw1602; 3: H5; 4: PSMA5; 5: Pda13015. Only HLA-A3101 was correctly amplified. 


\subsection{Test Reproducibility of HLA-A3101 on MBLF Strips}

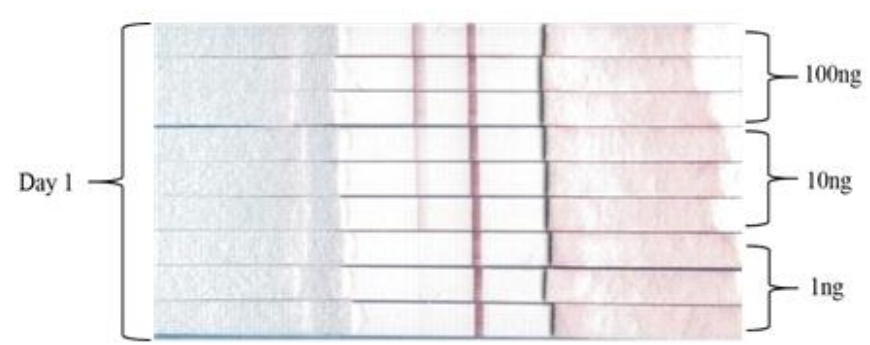

Fig. 9. The Intra-assay reproducibility test for HLA-A3101 on MBLF strips. Three amounts of samples, 100ng, 10ng, and 1ng of the PCR product were loaded in three replicate strips at the same time.

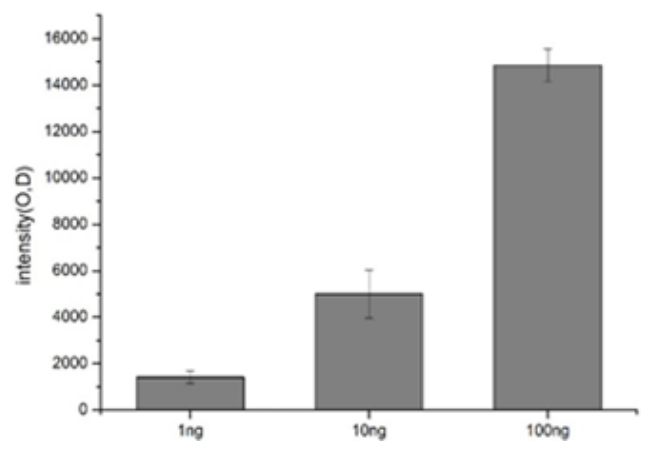

Fig. 10. The digitized plot of the Intra-assay reproducibility test for HLA-A3101 on MBLF strips.

The Inter-assay test run on MBLF strips and its digitized plot are also shown in Figures 11 and 12 , respectively. The statistic data shows the strip-to-strip (or day-to-day) variation keeps a good range within $6.3 \%$, indicating this detection approach gives a stable result for continual tests. It is very meaningful for a clinic to ensure a quick and reliable diagnosis always robustly provided by this detection method.

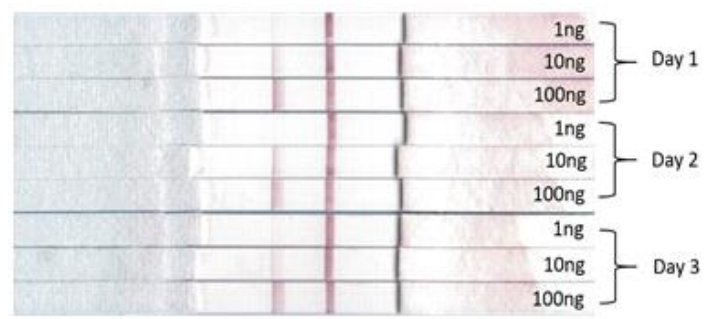

Fig. 11. The Inter-assay reproducibility test for HLA-A3101 on MBLF strips. Three amounts of samples, 100ng, 10ng, and 1ng of the PCR product were loaded in three consecutive days.

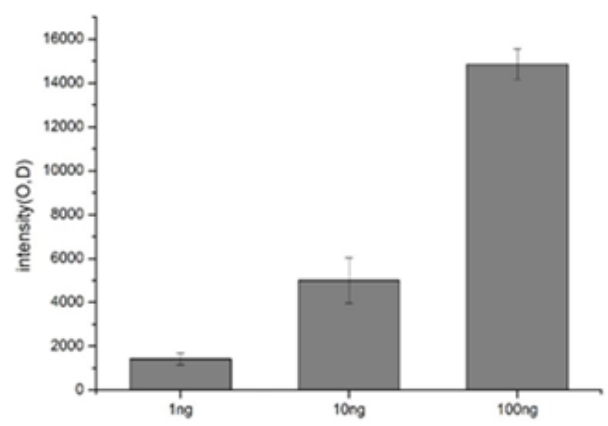

Fig. 12. The digitized plot of the Inter-assay reproducibility test for HLA-A3101 on MBLF strips.

The test reproducibility of HLA-A3101 on MBLF strips was conducted by loading 100ng, 10ng, and 1ng of 
its PCR product in two sets. For the Intra-assay test set, three replicates of each sample amount were run at the same day; while the Inter-assay test set repeated the same test in three consecutive days. The Intra-assay test run on MBLF strips and its digitized plot are shown in Figures 9 and 10, respectively. The statistical data shows the strip-to-strip variation keeps a good range within $4.6 \%$, indicating the reagent-dispensing process and pad-assembling procedure of MBLF strips had a low manipulation variation. As well, the test operator had a sophisticated skill, though operation training might not be necessary for the MBLF technology.

\section{Conclusion}

This study successfully amplified HLA-A3101 gene in optimized PCR condition and conducted rapid tests of the products on MBLF strips in 5 minutes. The readouts can be interpreted by naked eyes without gel electrophoresis and other instrument aids. The specification test indicates the HLA-A3101 primers gave an excellent loyalty to identify their own template from others. The reproducibility test indicates our samples were run in a stable experimental skill and the detection approach gave steady readouts.

\section{Acknowledgment}

This work was supported by a grant from the Department of Ministry of Science and Technology of Taiwan, Project MOST 106-2221-E-033-075.

\section{References}

[1] Gough, S., \& Simmonds, M. (2007) The HLA region and autoimmune disease: Associations and mechanisms of action. Current Genomics, 8, 453-465.

[2] Wang, Q., Mei, H., Zhang, Y., Pan, X., Tan, W., \& Chao, L. (2013). The associations between idiosyncratic adverse drug reactions and HLA alleles and their underlying mechanism. Acta Pharmaceutica Sinica, 799-808.

[3] Yip, V., Alfirevic, A., \& Pirmohamed, M. (2015). Genetics of immune-mediated adverse drug reactions: a comprehensive and clinical review. Clin Rev Allergy Immunol, 48(2-3), 165-175.

[4] Yang, Y., Yuan, L., Fang, X., Liang, X., \& Yang, F. (2013). Detection of HLA-B*27 gene using a spectral plasmon resonance imaging system. Biosens Bioelectron, 46, 80-83.

[5] Hung, S., Chung, W., Liou, L., Chu, C., Lin, M., Huang, H., \& Chen, Y. (2004). HLA-B*5801 allele as a genetic marker for severe cutaneous adverse reactions caused by allopurinol. Medical Sciences, 4134-4139.

[6] Jung, J., Song, W., Kim, Y., Joo, K., Lee, K., Kim, S., \& Kang, H. (2011). HLA-B5801 can help the clinical decision on starting allopurinol in patients with chronic renal insufficiency. Nephrology, dialysis, transplantation: Official publication of the European Dialysis and Transplant Association. European Renal Association, 26, 3567-3572.

[7] Shyu, R., Tang, S., Chiao, D., \& Hung, Y. (2010). Gold nanoparticle-based lateral flow assay for detection of staphylococcal enterotoxin B. Food Chem., 118, 462.

[8] Shyu, R., Shyu, H., Liu, H., \& Tang, S. (2002). Colloidal gold-based immunochromatographic assay for detection of ricin. Toxicon, 40, 255.

[9] Anfossia, L., Calderaraa, M., Baggiania, C., Giovannolia, C., \& Arletti, E. (2010). Development and application of a quantitative lateral flow immunoassay for fumonisins in maize. Anal. Chim. Acta, 682, 104.

[10] Wang, X., Li, K., Shi, D., Xiong, N., Jin, X., \& Yi, J. (2007). Development of an immunochromatographic lateral-flow test strip for rapid detection of sulfonamides in eggs and chicken muscles. Agr. and Food Chem., 55, 2072. 
[11] Tao, J., Zhong, L., Wei, R., \& Juan, C. (2011). Development and validation of a lateral flow immunoassay using colloidal gold for the identification of serotype-specific foot-and-mouth disease virus $0, \mathrm{~A}$ and Asia 1. Virological Methods, 171, 74.

[12] Ferris, P., Nordengrahnb, A., Hutchingsa, G., Patona, D., \& Kristerssonb, T. (2010). Development and laboratory validation of a lateral flow device for the detection of serotype SAT 2 foot-and-mouth disease viruses in clinical samples. Virological Methods, 163, 474.

[13] Schubert-Ullrich, P., Rudolf, J., Ansari, P., Galler, B., Führer, M., \& Molinelli, A. (2009). Commercialized rapid immunoanalytical tests for determination of allergenic food proteins: An overview. Anal. and Bioanal. Chem., 395, 69.

[14] Tsay, A., Williams, L., Mitchell, E., \& Chapman, M. (2002). Multi-Centre Study group. A rapid test for detection of mite allergens in homes. Clin. \& Exp. Allergy, 32, 1596.

[15] Horng, Y., Hsueh, P., Hsieh, S., Hung, Y., Lai, H., Lo, K., Shen, B., Soo, P., Su, H., \& Wei, J. (2006). Development of an improved PCR-ICT hybrid assay for direct detection of Legionellae and Legionella pneumophila from cooling tower water specimens. Water Research, 40, 2221-2229.

[16] Amerongen, A., Schallig, H., Mens, P., Sawa, P., \& Kager, P. (2008). Molecular diagnosis of malaria in the field: devel-opment of a novel 1-step nucleic acid lateral flow immunoassay for th-e detection of all 4 human Plasmodium spp. and its evaluation in Mbita,Kenya. Diagnostic Microbiology and Infectious Disease, 61, 421-427.

[17] Peng, D., Hu, S., Hua, Y., Xiao, Y., Li, Z., \& Wang, X. (2007). Comparison of a new gold-immunochromatographic assay for the detection of antibodies against avian influenza virus with hemagglutination inhibition and agar gel immunodiffusion assays. Immunol. and Immunop, 117, 17.

[18] Sharma, S., Eblen, B., Bull, R., Burr, D., \& Whiting, R. (2005). Evaluation of lateral-flow Clostridium botulinum neurotoxin detection kit for food analysis. Appl. Environ. Microbiol., 71, 3935.

[19] Diederen, B., \& Peeters, M. (2006). Evaluation of rapid U Legionella plus test, a new immunochromatographic assay for detection of Legionella pneumophila serogroup 1 antigen in urine. Clin. Microbiol. Infec. Dis., 25, 733.

[20] Wu, J., Chen, C., Fu, G., \& Yang, H. (2014). Electrophoresis-enhanced detection of deoxyribonucleic acids on a membrane-based lateral flow strip using Avian Influenza H5 genetic sequence as the model. Sensors, 14, 4399-4415.

[21] Han, Y., Liu, H., Zheng, H., Li, S., \& Bi, R. (2004). Purification and refolding of human $\alpha 5$-subunit (PSMA5) of the 20S proteasome, expressed as inclusion bodies in Escherichia coli. Protein Expr. Purif., 35, 360-365.

[22] Stephenson, \& Frank H. (2003) Calculations for Molecular Biology and Biotechnology (1st ed). California, USA: Academic Press, ch. 8, 151.

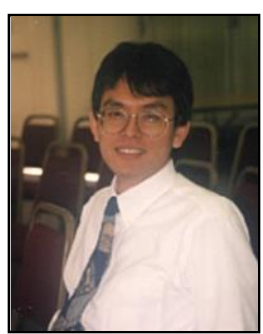

Jui-Chuang Wu worked in biotech industry in USA for 7 years with expertise in MBLF technology and biochips. He moved back to Taiwan and worked for 1 year at the Scientific Park in Hsin Chu city in the field of gene chips. He currently works as an associate professor in Chung Yuan Christian University, Taiwan, ROC.

Yu-Shiun Huang was a graduate school student at Chung Yuan Christian University. He stayed at Dr. Wu's lab to pursuit his master degree for 2 years. 